\title{
PAPER
}

\section{Central motor conduction in patients with anti-ganglioside antibody associated neuropathy syndromes and hyperreflexia}

\author{
Y Oshima, T Mitsui, H Yoshino, I Endo, M Kunishige, A Asano, T Matsumoto
}

J Neurol Neurosurg Psychiatry 2002;73:568-573

See end of article for authors' affiliations

......................

Correspondence: Dr T Mitsui, First

Department of Internal Medicine, School of

Medicine, The University of

Tokushima,

Kuramoto-3-18-15,

Tokushima 770-8503,

Japan;

tmitsui@

clin.med.tokushima-u.ac.jp

Received 29 January 2002

In revised form 2 July 2002

Accepted 31 July 2002

\begin{abstract}
Objectives: Several serum antibodies against gangliosides are diagnostically important, particularly in Guillain-Barré syndrome (GBS), Miller Fisher syndrome (MFS), and multifocal motor neuropathy (MMN). Although hyperreflexia is an atypical symptom in these disorders, it has been found in some patients with GBS, MFS, and MMN. The aim of the study was to determine whether hyperreflexia corresponds to corticospinal tract dysfunction in these patients.

Methods: The study examined central and peripheral motor conduction in patients with hyperreflexia who exhibited acute paralysis (group 1, $n=5$ ), acute ataxia and ophthalmoplegia (group 2, n=7), or chronic paralysis with conduction block (group $3, n=2$ ). The clinical symptoms are similar to those in patients with GBS, MFS, and $M M N$, respectively, and serum anti-ganglioside antibodies were found to be positive in all patients. Using magnetic and electrical stimulation techniques, central and peripheral motor conduction were compared in patients in groups 1, 2, and 3 and patients with GBS ( $n=7)$, MFS $(n=8)$, and $M M N(n=6)$.

Results: Central motor conduction times (CMCTs) in patients in groups 1, 2, and 3 were significantly delayed compared with those in patients with GBS, MFS, and MMN $(p<0.01, p<0.05, p<0.05$, respectively), and the delayed CMCTs significantly improved in the recovery periods $(p<0.01, p<0.01$, $p<0.05$, respectively). However, motor conduction velocity, compound muscle action potential, and $F$ wave conduction velocity were not significantly different between the patients.

Conclusion: These findings indicate that corticospinal tract is functionally involved in patients with anti-ganglioside antibody associated neuropathy syndromes and hyperreflexia
\end{abstract}

S everal antibodies against gangliosides have been detected n sera of patients with a variety of neuropathy syndromes. These antibodies seem to be associated with pathophysiological mechanisms of neuropathy syndromes, and some of them are diagnostically important, particularly in Guillain-Barré syndrome (GBS), Miller Fisher syndrome (MFS), and multifocal motor neuropathy (MMN). Sera from patients with GBS include several antibodies that react with human gangliosides of central and peripheral nervous systems, including GM1, GM2, GDla, GDlb, GQ1b, GalNAcGDla, and GMIb. ${ }^{1-4}$ GBS is an immune mediated polyradiculoneuropathy clinically characterised by acute generalised paralysis and areflexia. However, hyperreflexia or a brisk reflex has been observed in some patients with GBS. ${ }^{5-8}$ Serum IgG antibodies to the ganglioside GQIb were found to be strongly associated with MFS or GBS with ophthalmoplegia. ${ }^{90}$ MFS is characterised by the acute onset of ophthalmoplegia, ataxia, and areflexia and is considered to be a variant of GBS. ${ }^{11}$ On the other hand, some studies have shown that MFS involves the brainstem and has similarities to the brainstem encephalitis of Bickerstaff (BBE). ${ }^{12} 13$ Although the nosological position of $\mathrm{BBE}$ is still controversial, there are some patients with long tract signs as well as ophthalmoplegia and ataxia, ${ }^{12-14}$ and anti-GQlb antibodies have frequently been detected in patients with BBE. ${ }^{15}{ }^{16} \mathrm{MMN}$ is also an immune mediated motor neuropathy with slowly progressive asymmetrical weakness. Electrophysiological testing reveals a multifocal partial conduction block, the hallmark of MMN. High titres of serum IgM binding to GMl ganglioside frequently occur in patients with MMN. ${ }^{17}$ Patients with MMN usually show lower motor neuron signs and no upper motor neuron signs, including hyperreflexia. However, the reported patients sometimes showed preserved tendon reflexes or even, although rarely, brisk reflexes. ${ }^{18} 19$

In this context, patients with hyperreflexia have been excluded from clinical pictures of GBS, MFS, and MMN. They have been disregarded because of hyperreflexia, even if other clinical findings are similar to those of GBS, MFS, and MMN and serum anti-ganglioside antibodies are positive. We previously reported three patients who exhibited acute generalised paralysis with hyperreflexia. ${ }^{20}$ Two of them were seropositive for anti-GMl antibody. A preliminary study indicated that central nerve conduction time was prolonged in those patients. Recently, we have experienced other patients with hyperreflexia who exhibited acute paralysis, acute ataxia and ophthalmoplegia, or chronic paralysis with conduction block. In this study, we aimed to highlight the patients with hyperreflexia in whom serum anti-ganglioside antibodies are detected, and we found that central motor conduction was delayed in these patients compared with that in patients with GBS, MFS, or MMN.

\section{METHODS \\ Patients}

The subjects of this study were 14 patients with antiganglioside antibody associated neuropathy syndrome who had been selected from Japanese patients who had been

Abbreviations: GBS, Guillain-Barré syndrome; MFS, Miller Fisher syndrome; $M M N$, multfocal motor neuropathy; $C M C T$, central motor conduction time; MCV, motor conduction velocity; FWCV, F wave conduction velocity; CMAP, compound muscle action potential; AMAN, acute motor axonal neuropathy; BBE, brainstem encephalitis of Bickerstaff 
Table 1 Clinical features of patients with hyperreflexia and anti-gangioside antibodies and control patients with GBS, MFS, and MMN

\begin{tabular}{|c|c|c|c|c|c|c|c|}
\hline Patient & Age/sex & $\begin{array}{l}\text { Antecedent } \\
\text { infection }\end{array}$ & $\begin{array}{l}\text { Muscle } \\
\text { weakness }\end{array}$ & Ophthalmoplegia & Ataxia & $\begin{array}{l}\text { Cranial } \\
\text { neuropathy* }\end{array}$ & $\begin{array}{l}\text { Serum } \lg G / \lg M \text { antibodies to } \\
\text { gangliosides }\end{array}$ \\
\hline \multicolumn{8}{|c|}{ Group 1 (acute paralysis group) } \\
\hline 1 & $37 / M$ & Pharyngitis & + & - & - & - & $\mathrm{GM} 1 / \mathrm{GM} 2$ (lgG) \\
\hline 2 & $18 / M$ & Pharyngitis & + & - & - & - & GTla (lgG) \\
\hline 3 & $46 / M$ & Colitis & + & - & - & - & $\begin{array}{l}\text { GDla (lgG) } \\
\text { GMl/GDla/GTIb(lgM) }\end{array}$ \\
\hline 4 & $20 / M$ & None & + & - & - & - & GTIb (lgG) \\
\hline 5 & $51 / M$ & Colitis & + & - & - & - & GMI (lgG) \\
\hline \multicolumn{8}{|c|}{ Group 2 (acute ataxia and ophthalmoplegia group) } \\
\hline 6 & $48 / M$ & None & - & + & + & VII & GMI/GaINAc-GDla/GTlb (lgG) \\
\hline 7 & $41 / F$ & Pharyngitis & - & + & + & - & GQ1b (lgG) \\
\hline 8 & $21 / F$ & Colitis & - & + & + & - & GQ1b (lgG) \\
\hline 9 & $30 / F$ & None & - & + & + & - & GQ1b (lgG) \\
\hline 10 & $57 / F$ & Pharyngitis & - & + & + & - & GQ1b (lgG) \\
\hline 11 & $48 / F$ & None & - & + & + & - & GalNAc-GDla (lgG) \\
\hline 12 & $74 / F$ & None & - & + & + & - & GQ1b (lgG) \\
\hline \multicolumn{8}{|c|}{ Group 3 (chronic paralysis with conduction block group) } \\
\hline 13 & $57 / M$ & None & + & - & - & $|X-X| \mid$ & GMI/GDIb (lgM) \\
\hline 14 & $49 / M$ & None & + & - & - & - & GM1 (lgM) \\
\hline \multicolumn{8}{|l|}{ GBS } \\
\hline 1 & $33 / M$ & None & + & - & - & - & GM1 (lgG) \\
\hline 2 & $24 / F$ & Colitis & + & - & - & V, VII, IX-XII & GMIb/GDIb (lgG) \\
\hline 3 & $23 / M$ & Pharyngitis & + & - & - & - & GMI/GTIb (lgG) \\
\hline 4 & $14 / M$ & Colitis & + & - & - & - & GMI/GTIb (lgG) \\
\hline 5 & $46 / M$ & Pharyngitis & + & - & - & - & GM1b (lgG) \\
\hline 6 & $58 / \mathrm{F}$ & None & + & - & - & - & GM2 (lgG) \\
\hline 7 & $33 / F$ & Pharyngitis & + & - & - & - & GMIb/GDIb (lgG) \\
\hline \multicolumn{8}{|r|}{ | } \\
\hline 1 & $73 / F$ & Pharyngitis & - & + & + & - & GQ1b (lgG) \\
\hline 2 & $60 / F$ & Pharyngitis & - & + & + & - & GM1/GM2/LM1 (lgG) \\
\hline 3 & $32 / F$ & Pharyngitis & - & + & + & - & GQ1b (lgG) \\
\hline 4 & $74 / M$ & None & - & + & + & - & GMI (lgM) \\
\hline 5 & $75 / F$ & Pharyngitis & - & + & + & - & GQ1b/GTla (lgG) \\
\hline 6 & $59 / \mathrm{F}$ & Pharyngitis & - & + & + & - & - \\
\hline 7 & $80 / \mathrm{F}$ & Pharyngitis & - & + & + & - & GQ1b (lgG) \\
\hline 8 & $25 / M$ & Pharyngitis & - & + & + & - & GQIb/GTla (lgG) \\
\hline \multicolumn{8}{|l|}{$M M N$} \\
\hline 1 & $51 / M$ & None & + & - & - & - & GM1 (IgM) \\
\hline 2 & $63 / F$ & None & + & - & - & - & - \\
\hline 3 & $43 / F$ & None & + & - & - & - & GMI/GDIb (lgM) \\
\hline 4 & $66 / M$ & None & + & - & - & - & GMI (IgM) \\
\hline 5 & $51 / \mathrm{F}$ & None & + & - & - & - & GMI (lgM) \\
\hline 6 & $58 / \mathrm{F}$ & None & + & - & - & - & - \\
\hline
\end{tabular}

treated at Tokushima University Hospital or its affiliated hospitals between January 1997 and May 2001 and had agreed to participate in this study. The 14 patients were classified into three groups according to the clinical pictures (table 1). Patients with disorders that can cause peripheral neuropathy were excluded by means of appropriate laboratory tests. All of the patients in groups 1,2, and 3 showed hyperreflexia in four limbs but not in jaw jerk at the first visit to hospital. MRI studies were performed throughout the central nervous system and revealed no compressive lesions in the spinal cord of any of the patients. In all patients, the neurological symptoms responded to plasma exchange (a total of 120 to $200 \mathrm{ml}$ of plasma per $\mathrm{kg}$ being removed in three treatment sessions) and/or intravenous immunoglobulin therapy (0.4 $\mathrm{g} / \mathrm{kg}$ for five consecutive days). In patients in groups 1 and 2, clinical symptoms completely recovered within several months. Clinical data of patients 1 and 3 were previously reported. ${ }^{20}$ Group 1 (acute paralysis group) consisted of five patients who exhibited symmetric muscle weakness with acute onset in all four limbs. The weakness ascended from the thighs to the arms and progressed for a week to four weeks. Hyperreflexia was observed in all four limbs during the progression stage in all patients but Babinski's sign was not observed in any of the patients. Cerebrospinal fluid (CSF) showed an increased concentration of protein (61.25 (17.7) $\mathrm{mg} / \mathrm{dl}$, mean (SD)) without pleocytosis. Electrophysiological testing demonstrated axonal neuropathy compatible with the criteria of Hadden $e a^{21}$ in all patients. Group 2 (acute ataxia and ophthalmoplegia group) consisted of seven patients who all had acute ataxia and ophthalmoplegia with or without other cranial neuropathies and with clinically insignificant sensory or motor deficits. Hyperreflexia was observed in all four limbs during the progression stage in all patients but Babinski's sign was not observed an any of the patients. The mean (SD) protein concentration in CSF was 41.4 (12.1) $\mathrm{mg} / \mathrm{dl}$ and there was not pleocytosis. Group 3 (chronic paralysis with conduction block group) consisted of two patients who showed slowly progressive, asymmetric limb weakness without sensory impairment. Nerve conduction study revealed persistent partial conduction block in at least two motor nerves. Hyperreflexia was observed in both patients and Babinski's sign was positive in one patients (patient 13). The protein concentration in CSF were mildly raised (54 and 65 $\mathrm{mg} / \mathrm{dl}$ ) and there was no pleocytosis.

Control patients with GBS $(n=7)$, MFS $(n=8)$, or MMN $(n=6)$ (table 1) were enrolled in this study for comparison with patients in groups 1,2, and 3, respectively. Because all of the patients in group 1 showed axonal motor neuropathy, the control patients were selected from patients with acute motor axonal neuropathy (AMAN) who fulfilled the criteria. ${ }^{21}$ MFS 
Table 2 Clinical disability scales before and after treatment

\begin{tabular}{|c|c|c|c|c|c|c|c|c|c|}
\hline & \multicolumn{3}{|c|}{ Modified Rankin disability scale* } & \multicolumn{3}{|c|}{ Greatest overall disability* } & \multicolumn{3}{|c|}{ Greatest arm weakness* } \\
\hline & $\begin{array}{l}\text { Before } \\
\text { treatment }\end{array}$ & After treatment $\dagger$ & $\mathrm{p} \ddagger$ & $\begin{array}{l}\text { Before } \\
\text { treatment }\end{array}$ & After treatment† & $\mathrm{p} \ddagger$ & $\begin{array}{l}\text { Before } \\
\text { treatment }\end{array}$ & After treatment $\dagger$ & $p \ddagger$ \\
\hline Group 1 & $3.2(1.47)$ & $1.4(1.36)$ & $p<0.001$ & $2.6(1.20)$ & $1.4(1.36)$ & $p<0.005$ & $2.2(0.75)$ & $1.0(0.89)$ & $p<0.005$ \\
\hline GBS & $3.4(1.40)$ & $0.9(0.64)$ & $p<0.001$ & $3.1(1.25)$ & $0.9(0.64)$ & $p<0.001$ & $2.3(1.05)$ & $0.9(0.64)$ & $p<0.005$ \\
\hline Group 2 & $2.6(0.73)$ & $1.0(0.53)$ & $p<0.005$ & $2.6(0.73)$ & $1.0(0.53)$ & $p<0.001$ & & & \\
\hline MFS & $2.9(1.27)$ & $1.3(1.09)$ & $p<0.001$ & $2.8(1.09)$ & $1.4(1.32)$ & $p<0.001$ & & & \\
\hline Group 3 & $3.0(1.0)$ & $2.5(1.0)$ & $p>0.1$ & $2.5(0.5)$ & $2.5(0.5)$ & $p=1$ & $2.0(1.0)$ & $1.0(1.0)$ & $p>0.1$ \\
\hline$M M N$ & $2.0(0.82)$ & $1.0(0.82)$ & $p<0.05$ & $2.0(0.82)$ & $1.3(1.11)$ & $p<0.05$ & $1.0(0)$ & $0.8(0.37)$ & $p>0.1$ \\
\hline
\end{tabular}

was defined as a clinical syndrome comprising ataxia with rapid onset, areflexia, and ophthalmoplegia with clinically insignificant sensory or motor deficits in addition to increased CSF protein without pleocytosis and favourable outcome. The diagnosis of MMN was based on the presence of slowly progressive, asymmetric limb weakness without sensory impairment, and persistent partial conduction block in at least two motor nerves. Patients with MMN exhibited areflexia in diseased areas but no hyperreflexia in four limbs. The clinical pictures are compatible with the MMN diagnostic assessment described by Pestronk. ${ }^{22}$ During the period from 1997 to 2001, 29 patients with demyelinating GBS, 12 patients with AMAN, 13 patients with MFS, and eight patients with MMN were treated in our hospitals. Patients with AMAN, MFS, and MMN who had agreed to participate in the study were enrolled as control patients after obtaining written informed consent.

\section{Clinical examinations}

Patients were assessed before and every week after treatment using (a) a modified Rankin disability scale ${ }^{23}$ (0 to 5): $0=$ asymptomatic, $\mathrm{l}=$ non-disabling symptoms not interfering with lifestyle, 2 =minor disability symptoms leading to some restriction of lifestyle but not interfering with the patient's capacity to look after themselves, $3=$ moderate disability symptoms significantly interfering with lifestyle or preventing fully independent existence, $4=$ moderately severe disability symptoms preventing independent existence although patients do not need constant attention day and night, $5=$ totally dependent, requiring constant attention day and night; (b) greatest overall disability ${ }^{24}(0$ to 5$)$ : $0=$ healthy, $\mathrm{l}=$ minor symptoms or signs and capable of running, $2=$ able to walk five metres across an open space without assistance, walking frame, or stick but unable to run, $3=$ able to walk five metres across an open space with the help of one person and waist level walking frame, stick or sticks, $4=$ chairbound or bed bound, unable to walk as in 3,5=assisted ventilation required (for at least part of the day); (c) greatest arm weakness ${ }^{23}$ ( 0 to 4): $0=$ normal, $1=$ minor symptoms or signs but able to put hand on top of head when sitting upright and able to oppose thumb to each fingertip, $2=$ either able to put hand on top of head when sitting upright or able to oppose thumb to each fingertip, but not both, $3=$ some movement but unable to do either of the tasks in 2,4=no movement. As patients in group 2 and patients with MFS did not exhibit significant weakness, the ataxia was estimated by scales (a) and (b).

\section{Anti-ganglioside antibodies}

Serum samples from all patients were screened by thin layer chromatography (TLC) and immunostaining using bovine brain gangliosides and acidic glycolipids of human peripheral nerves, as described elsewhere. ${ }^{1}$ These lipids contain several gangliosides such as GM1, GM2, GM3, GDla, GD3, GQ1b, GalMAc-GDla, GTla, GD2, GDlb, GTlb, LM1, Hex-LM1,
SGPG, and SGLPG. The immunopositive sera were also examined by the enzyme linked immunosorbent assay for both IgG and IgM antibodies as described in our previous reports. ${ }^{32}$ In brief, after $10 \mathrm{pmol}$ of each ganglioside had been placed in wells, serum samples diluted 1:100 were added. A peroxidaseconjugated antihuman $\gamma$ or $\mu$ chain specific antibody was used, and absorbance values at $\mathrm{OD}_{492}$ were obtained by subtracting the respective values of wells without antigen.

\section{Electrophysiological studies}

Nerve conduction studies were performed in all patients before treatment and at one, two, and four weeks after treatment. Motor conduction was examined in bilateral median, ulnar, peroneal, and tibial nerves according to routine procedures. Distal latencies, conduction velocity, and compound muscle action potential (CMAP) were measured. For the detection of conduction block, median and ulnar nerves were stimulated at the wrist, elbow and Erb's point; the peroneal nerve at the ankle and below and above the fibular head; and the tibial nerve at the ankle and popliteal fossa. Partial motor conduction block was defined as a reduction in the ratio of proximal to distal CMAP amplitude to $<0.60$ only when the ratio of proximal to distal negative peak duration was $<1.15$ and distal amplitude exceeded $1 \mathrm{mV}$. Latencies for $\mathrm{F}$ waves were measured after distal supramaximal stimulation (at least 16 stimuli) in the median and ulnar nerves. Sensory conduction was examined in median, ulnar, and sural nerves.

\section{Magnetic stimulation study}

Magnetic stimulation was performed as previously described..$^{20}$ In brief, motor evoked potentials (MEPs) were stimulated using a Magnestim Model 200 (Magnestim Company, Wales, UK) and were recorded with surface electrodes from bilateral abductor pollicic brevis. MEP latencies were measured to the first negative deflection of the potential, and repetitive examinations (at least three times) confirmed the reliability of the latencies. Central motor conduction time (CMCT) was calculated by subtracting $(\mathrm{F}+\mathrm{M}-\mathrm{l}) / 2$ from the corresponding MEP latency. The peripheral component, $\mathrm{F}$ and $\mathrm{M}$ waves were obtained by electrical stimulation of the median nerve and were recorded with surface electrodes from bilateral abductor pollicis brevis.

\section{Statistical analysis}

We used StatView for Windows (version 5.0) for statistical analysis. Non-paired data were analysed by the nonparametric Mann-Whitney U test, and paired data were analysed by Wilcoxon's signed rank test.

\section{RESULTS}

\section{Clinical assessment}

Patients in groups 1 and 2 underwent plasma exchange and/or intravenous immunoglobulin therapy, and patients in group 3 

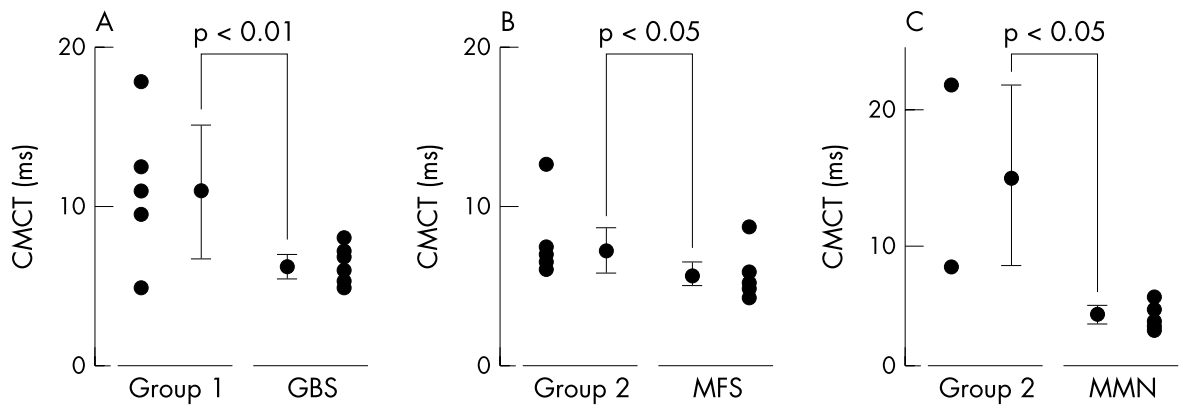

Figure 1 Central motor conduction time (CMCT) in test patients and control patients. CMCT is significantly prolonged in patients in groups 1 , 2, and 3 than in patients with GBS, MFS, and MMN, respectively. (A) Group 1 (acute paralysis group) compared with GBS. (B) Group 2 (acute ataxia and ophthalmoplegia group) compared with MFS. (C) Group 3 (chronic paralysis with conduction block group) compared with MMN.
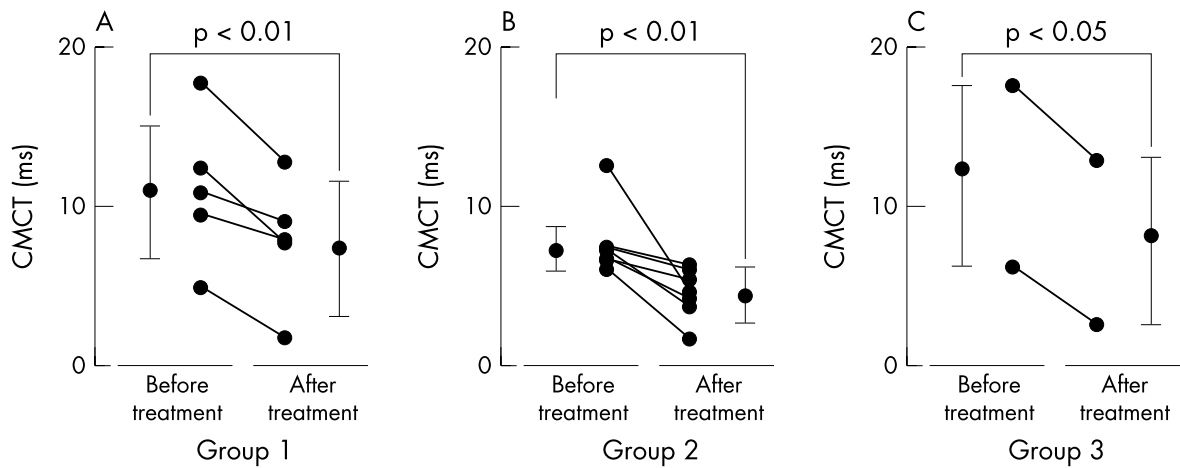

Figure 2 Central motor conduction time (CMCT) before and after treatment in patient in groups $1(\mathrm{~A}), 2(\mathrm{~B})$, and $3(\mathrm{C})$. CMCT is significantly improved two to three weeks after the initial treatment in patients in all three groups.

underwent intravenous immunoglobulin therapy. The following three disability scales were used to assess clinical symptoms: modified Rankin disability scale, greatest overall disability scale, and greatest arm weakness scale. As shown in table 2, the clinical disability scores of patients were not significantly different from those of the respective controls $(p>0.1)$. The scores in each of the scales significantly decreased after treatment in patients in groups 1 and 2. After treatment, the scores in the modified Rankin disability scale, greatest overall disability scale and greatest arm weakness scale were not significantly different between patients in groups 1 and patients with GBS (AMAN) $(p>0.1)$ and between patients in group 2 and patients with MMN $(p>0.1)$, indicating that patients in group 1 and 2 had similar clinical disabilities and therapeutic responses to those of patients with GBS(AMAN) and MFS, respectively. As group 3 consisted of only two patients, the scores in these scales were not significantly improved $(p>0.1)$. In patients with $M M N$, the scores in the modified Rankin disability scale and the greatest overall disability scale, but not the scores in greatest arm weakness scale, were significantly decreased after intravenous immunoglobulin therapy $(\mathrm{p}<0.05)$. Hyperreflexia was present at the start of treatment in all patients in groups 1, 2, and 3, and it slowly improved. In patients in groups 1 and 2, hyperreflexia persisted until two to six months after clinical disabilities had completely disappeared. In patients in group 3 , hyperreflexia did not improve after treatment.

\section{Anti-ganglioside antibodies}

As shown in table 1 , patients in group 1 (acute paralysis group) showed various anti-ganglioside IgG antibodies in their sera, and the anti-GMl IgG antibody was detected in two patients (patients 1 and 5). IgM antibodies were detected only in patient 3. The anti-GQlb IgG antibody was detected in five of seven patients in group 2 (acute ataxia and ophthalmople- gia group). High titres of the anti-GMI IgM antibody was observed in both patients in group 3 (chronic paralysis with conduction block group), and patient 13 showed an additional IgM antibody against GDlb.

\section{Central and peripheral motor conduction}

Figures 1 and 2 show central motor conduction via the right median nerve. CMCT in group 1 patients was 11.1 (4.7) $\mathrm{ms}$ (mean (SD)), which was significantly delayed compared with that in patients with GBS (AMAN) $(5.4$ (1.1) ms, p $<0.01$, fig lA). However, motor conduction velocity (MCV), CMAP, and FWCV of the right median nerve were not significantly different from those in patients with GBS (AMAN) ( $p>0.5$, not shown). Figure 3 shows representative profiles of MEPs, $M$ waves, and $\mathrm{F}$ waves in one patient in group 1. MEP latency was delayed before plasma exchange and had recovered four weeks after the treatment. However, latencies of $\mathrm{M}$ waves and $\mathrm{F}$ waves were not prolonged and did not change after the treatment. CMCTs in group 2 patients (fig $1 \mathrm{~B}$ ) and group 3 patients (fig 1C) were 7.7 (1.5) and 15.4 (7.2) ms, respectively, which were significantly delayed compared with those in patients with MFS (6.3 (1.3) ms, p<0.05) and MMN (4.8 (1.3) ms, p<0.05). MCV, CMAP, and FWCV of bilateral median nerves in patients of groups 2 and 3 were not significantly different from those in patients with MFS and MMN ( $p>0.5)$. Selective delays in CMCT were also observed in patients in groups 1,2 , and 3 at contralateral stimulation $(p<0.01$, $\mathrm{p}<0.05, \mathrm{p}<0.05$, respectively). As the clinical symptoms of the patients improved after plasma exchange and/or intravenous immunoglobulin therapy, we next examined whether the delayed CMCT recovered after treatment. As shown in figure 2, CMCT significantly improved two to three weeks after the initial treatment in patients in group 1 (7.3 (4.3) ms, p<0.01), group $2(4.5(2.0) \mathrm{ms}, \mathrm{p}<0.01)$, and group $3(8.1$ (6.1) $\mathrm{ms}$, $\mathrm{p}<0.05)$. The delays in CMCT also improved in patients in 
MEP

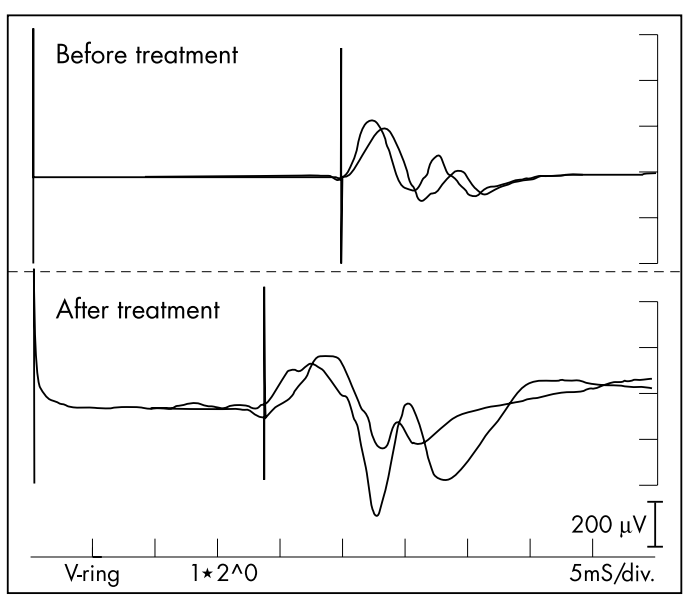

F wave

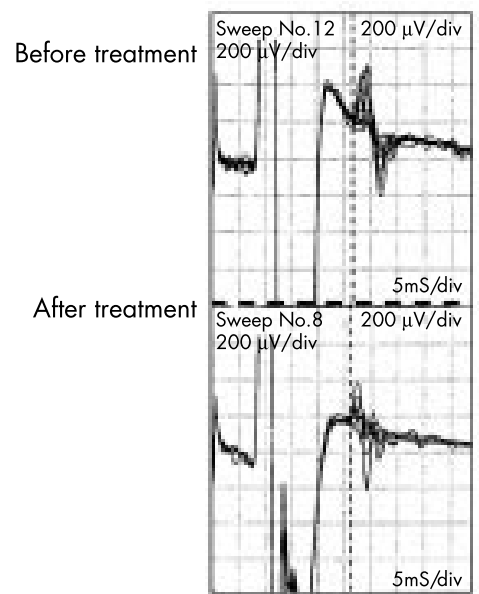

Figure 3 Representative profiles of motor evoked potentials (MEPs), M wave and F waves in a patient in group 1. MEP latency was delayed before plasma exchange and recovered four weeks after the treatment. However, latencies of $M$ waves and $F$ waves were not prolonged and not changed after the treatment.

groups 1,2 , and 3 at contralateral stimulation $(\mathrm{p}<0.01$, $\mathrm{p}<0.01, \mathrm{p}<0.05$, respectively).

\section{DISCUSSION}

In this study, we investigated 14 patients with hyperreflexia who exhibited acute paralysis, acute ataxia, and ophthalmoplegia or chronic paralysis with conduction block. The presence of serum anti-ganglioside antibodies, but not the presence of hyperreflexia, was compatible with GBS, MFS, or MMN. Hyperreflexia is actually a negative symptom in GBS and MFS. We therefore recognised these patients as antiganglioside antibody associated neuropathy syndromes. To determine whether hyperreflexia corresponds to corticospinal tract dysfunction, we evaluated central motor conduction using a magnetic stimulation technique, the diagnostic value of which has been applied to a wide variety of central nervous system disorders. In particular, CMCT is delayed according to upper motor neuron involvement in patients with motor neuron disease. ${ }^{2627}$ We compared central and peripheral motor conduction in patients in groups 1,2, and 3 and patients with GBS (AMAN), MFS, and MMN, respectively. The scores in disability scales were not significantly different in patients and respective control patients either before or after treatment, indicating that the clinical courses of the patients with hyperreflexia were similar to those of the control patients. On the other hand, CMCT was selectively delayed in patients with hyperreflexia, and clinical improvement in those patients was followed by improvement in CMCT. Hyperreflexia is not caused only by corticospinal tract dysfunction and it is possible that other supraspinal influences are involved in the development of hyperreflexia in the present patients. However, our results at least indicate that the corticospinal tract is functionally impaired in patients with hyperreflexia. Because MEPs were recorded from the upper limbs in this study, corticomotoneuronal involvement seems to exist above the cervical segment and to be associated with the pathophysiological nature of anti-ganglioside antibody associated neuropathy syndromes.

By the criteria of GBS, patients with hyperreflexia are principally excluded from the syndrome. ${ }^{28}$ However, hyperreflexia or brisk tendon reflexia has been observed in some patients with generalised paralysis with antecedent infection..$^{5-8}$ Electrophysiological study showed motor axonal neuropathy, similar to AMAN, a variant of $\mathrm{GBS}^{7}{ }^{7}$ These findings are consistent with the present cases in group 1. Deep tendon reflexes seem to have been preserved in AMAN patients with a mild clinical course in northern China, probably resulting from the clinical course that areflexia recovers more quickly than flaccid paresis. ${ }^{6-8}$ On the other hand, increased soleus H-reflex amplitudes and the appearance of hand H-reflexes have been found in patients with AMAN and hyperreflexia, indicating increased motor neuron excitability. ${ }^{7}$ A necropsy study of AMAN demonstrated Wallerian-like degeneration of motor fibres and intensive involvement in the spinal cord. ${ }^{29}$ Swollen and rounded motor neurons with chromatolytic nuclei have been detected in anterior horn cells and in medial and lateral motor neuron populations. ${ }^{29}$ The pathological features are in sharp contrast with those of demyelinating GBS as central nervous systems are generally intact except for posterior column degeneration in the spinal cord. ${ }^{50}$ These findings are concordant with the present results that hyperreflexia was observed only in patients with AMAN and that CMCT was not delayed in nine patients with demyelinating GBS (data not shown). Although the clinical significance is still controversial and upper motor neuron involvement in patients with AMAN has not been established, the results of this study indicate the dysfunction of corticospinal tract in the patients with anti-ganglioside antibody associated neuropathy syndrome, corresponding to hyperreflexia.

MFS is considered to be a variant of GBS because of the disturbances of peripheral nervous systems. However, the relation has been the subject of much debate, because MFS involves brainstem lesions and bears similarities to BBE. ${ }^{12-14} 31$ Serum antibodies against GQIb have been significantly detected in patients with MFS as well as with BBE, indicating a common autoimmune mechanism. ${ }^{910}{ }^{15}{ }^{16}$ Although areflexia is a hallmark of MFS, brisk tendon reflexes have been reported in patients with MFS. ${ }^{12-14}$ This may depend on the nosological position of BBE, which is still controversial. Seven patients with hyperreflexia who exhibited acute ataxia and ophthalmoplegia were included in this study. We assigned these patients to group 2, and we investigated the central and peripheral motor conduction. Peripheral motor conduction was found to be normal in all patients in group 2, but central motor conduction time was significantly prolonged compared with that in patients with MFS. These findings suggest that the corticospinal tract is potentially impaired in patients with acute ataxia and ophthalmoplegia, associated with anti-GQIb antibody.

MMN is clinically characterised by a chronic, asymmetric, pure motor neuropathy associated persistent multifocal partial conduction block. High concentrations of serum anti-GMl antibodies have often been found in patients with MMN. ${ }^{17-19}$ Deep tendon reflexes are usually reduced or normal, 
but brisk reflexes are rarely observed..$^{19}$ The diagnosis of MMN is mainly based on electrophysiological findings, and MMN does not show common pathological features, including normal or inconsistent findings in biopsied sural nerve. $^{18}{ }^{19}{ }^{32-35}$ Furthermore, little is known about pathological changes in the central nervous systems. This study showed that CMCT was selectively delayed in patients with hyperreflexia and chronic paralysis with conduction block. Although group 3 consisted of only two patients, we found delayed CMCT in an additional two patients with similar clinical symptoms in whom anti-ganglioside antibodies were not detected (data not shown). These findings indicate that the corticospinal tract is at least functionally involved in some patients with MMN and hyperreflexia.

\section{Authors' affiliations}

Y Oshima, T Mitsui, I Endo, M Kunishige, T Matsumoto, First Department of Internal Medicine, School of Medicine, The University of Tokushima, Tokushima, Japan

H Yoshino, A Asano, Department of Neurology, Kohnodai Hospital, National Centre of Neurology and Psychiatry, Chiba, Japan

Competing interests: none declared.

\section{REFERENCES}

1 Ilyas AA, Willson HJ, Quarles RH, et al. Serum antibodies to gangliosides in Guillain-Barré syndrome. Ann Neurol 1988;23:440-7.

2 Kuwabara S, Yuki N, Koga M, et al. IgG anti-GMI antibody is associated with reversible conduction failure and axonal degeneration in Guillain-Barré syndrome. Ann Neurol 1998;44:202-8.

3 Hao Q Saida T, Yoshino $\mathrm{H}$, et al. Anti-GalNAc-GDla antibody-associated Guillain-Barré syndrome with a predominantly distal weakness without cranial nerve impairment and sensory disturbance. Ann Neurol 1999;45:758-68.

4 Rees JH, Gregson NA, Hughes RAC. Anti-ganglioside GMI antibodies in Guillain-Barré syndrome and their relationship to campylobacter jejun infection. Ann Neurol 1995;38:809-16.

5 Ropper AH. The Guillain-Barré syndrome. N Engl J Med 1992;326:1130-6.

6 MaKhann GM, Cornblath DR, Ho T, et al. Clinical and electrophysiological aspects of acute paralytic disease of children and young adults in northern China. Lancet 1991;338:593-7.

7 Kuwabara S, Ogawara K, Koga M, et al. Hyperreflexia in Guillain-Barré syndrome: relation with acute motor axonal neuropathy and anti-GM1 antibody. J Neurol Neurosurg Psychiatry 1999;67:180-4.

8 Yuki N, Hirata K. Preserved tendon reflexes in campylobacter neuropathy. Ann Neurol 1998;43:546-7.

9 Chiba A, Kusunoki S, Obata H, et al. Serum anti-GQ1b lgG antibody is associated with ophtalmoplegia in Miller Fisher syndrome and Guillain-Barré syndrome. Neurology 1993:43:1911-17.

10 Willison HJ, Veitch J, Paterson G, et al. Miller Fisher syndrome is associated with serum antibodies to GQ1b ganglioside. J Neurol Neurosurg Psychiatry 1993;56:204-6.

11 Fisher M. An unusual variant of acute idiopathic polyneuritis (syndrome of ophthalmoplegia, ataxia and areflexia) N Engl J Med 1956;255:57-65.
12 Al-Din AN, Anderson M, Bickerstaff ER, et al. Brainstem encephalitis and the syndrome of Miller Fishers. A clinical study. Brain 1982; 105:481-95.

13 Petty RKH, Duncan R, Jamal GA, et al. Brainstem encephalitis and the Miller Fisher syndrome. J Neurol Neurosurg Psychiatry 1993; 56:201-3.

14 Miwa H, Imamura N, Kogahara K, et al. Somatosensory evoked blink response: findings in patients with Miller Fisher syndrome and in normal subjects. J Neurol Neurosurg Psychiatry 1995;58:95-9.

15 Yuki N, Sato S, Tsuji S, et al. An immunologic abnormality common to Bickerstaff's brain stem encephalitis and Fisher's syndrome. J Neurol Sci 1993;1 18:83-7.

16 Chiba A, Kusunoki S, Obata H, et al. Ganglioside composition of the human cranial nerves, with special reference to pathophysiology of Miller Fisher syndrome. Brain Res 1997;745:32-6.

17 Pestronk A, Choksi R. Multifocal motor neuropathy. Serum IgM anti-GM1 ganglioside antibodies in most patients detected using covalent linkage of GM1 to ELISA plates. Neurology 1997;49:1289-92.

18 Pestronk A, Cornblath DR, llyas AA, et al. A treatable multifocal motor neuropathy with antibodies to GMI ganglioside. Ann Neurol 1988;24:73-8.

19 Nobile-Orazio E. Multifocal motor neuropathy. J Neurol Neurosurg Psychiatry 1996;60:599-603

20 Oshima Y, Mitsui T, Endo I, et al. Corticospinal tract involvement in a variant of Guillain-Barré syndrome. Eur Neurol 2001:46:39-42.

21 Hadden RDM, Cornblath DR, Hughes RAC, et al. Electrophysiological classification of Guillain-Barré syndrome: clinical associations and outcome. Ann Neurol 1998;44:780-8.

22 Pestronk A. Multifocal motor neuropathy: diagnosis and treatment. Neurology 1998;51(suppl 5):S22-4.

23 Nobile-Orazio E, Meucci N, Barbieri S, et al. High dose intravenous immunoglobulin therapy in multifocal motor neuropathy. Neurology 1993;43:537-44

24 Guillain-Barré syndrome steroid trial group. Double-blind trial of intravenous methylpredonisolone in Guillain-Barré syndrome. Lancet 1993;341:586-90.

25 Yoshino H, Harukawa $H$, Asano A. Ig G antiganglioside antibodies in Guillain-Barré syndrome with bulbar palsy. J Neuroimmunol 2000; 105: 195-201.

26 Schulte-Mattler WJ, Müller T, Zierz S. Transcranial magnetic stimulation compared with upper motor neuron signs in patients with amyotrophic lateral sclerosis. J Neurol Sci 1999;170:51-6.

27 Molinuevo JL, Cruz-Martínez A, Graus F, et al. Central motor conduction time in patients with multifocal motor conduction block. Muscle Nerve 1999:22:926-32.

28 Asbury AK, Cornblath DR. Assesment of current diagnotic criteria for Guillain-Barré syndrome. Ann Neurol 1990;27 (suppl):S2 1-4.

29 MaKhann GM, Cornblath DR, Griffin JW, et al. Acute motor axonal neuropathy : a frequent cause of acute flaccid paralysis in China. Ann Neurol 1993;33:333-42

30 Honavar M, Tharakan JKJ, Hughes RAC, et al. A clinicopathological study of the Guillain-Barré syndrome. Brain 1991;114:1245-69.

31 Ferrer $\mathbf{X}$, Ellie $E$, Larriviere $M$, et al. Late central demyelination after Fisher's syndrome: MRI studies. J Neurol Neurosurg Psychiatry 1993:56:693-9.

32 Krarup C, Stewart JD, Sumner AJ. A syndrome of aymmetric limb weakness with motor conduction block. Neurology 1990;40:1 18-27.

33 Bouche P, Moulonguer A, Younes-Chennoufi AB, et al. Multifocal motor neuropathy with conduction block: a study of 24 patients. J Neurol Neurosurg Psychiatry 1995;59:38-44.

34 Kornberg AJ, Pestronk A. Chronic motor neuropathies: diagnosis, therapy and pathogenesis. Ann Neurol 1995;37 (suppl 1):S43-50.

35 Corse AM, Chaudhry V, Crawford TO, et al. Sensory nerve pathology in multifocal motor neuropathy. Ann Neurol 1996;39:319-25. 